\title{
Magnetized Neutron-Star Mergers and Gravitational-Wave Signals
}

\author{
Matthew Anderson, ${ }^{1}$ Eric W. Hirschmann, ${ }^{2}$ Luis Lehner, ${ }^{3}$ Steven L. Liebling, ${ }^{4}$ Patrick M. Motl, ${ }^{3}$ David Neilsen, ${ }^{2}$ \\ Carlos Palenzuela, ${ }^{3,5}$ and Joel E. Tohline ${ }^{3}$ \\ ${ }^{1}$ Department of Mathematics, Brigham Young University, Provo, Utah 84602, USA \\ ${ }^{2}$ Department of Physics and Astronomy, Brigham Young University, Provo, Utah 84602, USA \\ ${ }^{3}$ Department of Physics and Astronomy, Louisiana State University, Baton Rouge, Louisiana 70803-4001, USA \\ ${ }^{4}$ Department of Physics, Long Island University-C.W. Post Campus, Brookville, New York 11548, USA \\ ${ }^{5}$ Max-Planck-Institut für Gravitationsphysik, Albert-Einstein-Institut, Golm, Germany
}

(Received 28 March 2008; published 15 May 2008)

\begin{abstract}
We investigate the influence of magnetic fields upon the dynamics of, and resulting gravitational waves from, a binary neutron-star merger in full general relativity coupled to ideal magnetohydrodynamics. We consider two merger scenarios: one where the stars have aligned poloidal magnetic fields and one without. Both mergers result in a strongly differentially rotating object. In comparison to the nonmagnetized scenario, the aligned magnetic fields delay the full merger of the stars. During and after merger we observe phenomena driven by the magnetic field, including Kelvin-Helmholtz instabilities in shear layers, winding of the field lines, and transition from poloidal to toroidal magnetic fields. These effects not only mediate the production of electromagnetic radiation, but also can have a strong influence on the gravitational waves. Thus, there are promising prospects for studying such systems with both types of waves.
\end{abstract}

DOI: 10.1103/PhysRevLett.100.191101

PACS numbers: 04.30.Db, 04.25.dk

I. Introduction. - To the long list of spectacular electromagnetic signals which researchers have employed to study our Universe, a new counterpart of gravitational origin will soon be added. Gravitational waves will both complement our ability to probe a variety of sources and in many cases provide the only option. These waves will likely be detected in the near future by a new generation of laser interferometers [1-3]. Among their most promising sources are merging binaries composed of black holes or neutron stars. These waves carry information about the dynamic, strong gravitational field near the binary. When one member of the binary is a neutron star, these waves can have a richer structure and carry additional information about the matter. For example, analysis of such mergers can potentially yield constraints on the nuclear equation of state and, as we show here, key insights on the stars' magnetic fields. The combination of gravitational-wave data with other astronomical observations in the electromagnetic spectrum will provide an enhanced understanding of many energetic, strongly gravitating phenomena, such as (short) gamma ray bursts.

Precise theoretical models are required to extract information from gravitational waves. Recently, significant progress has been made in modeling the radiation expected from binary black holes, binary neutron stars, and blackhole-neutron-star binaries. However, much less is known about how magnetic fields may alter the dynamics of such mergers, whose effects would be encoded in the gravitational waveforms. Magnetic fields are present in many astrophysical systems, and are important in several interesting phenomena, such as active galactic nuclei, pulsars, gamma ray bursts, etc. Observational evidence indicates that neutron stars have some of the strongest known mag- netic fields, with estimated field strengths ranging from $10^{12} \mathrm{G}$ to as large as $10^{16} \mathrm{G}$ in magnetars [4]. Magnetic field effects in merging or collapsing scenarios have been investigated in related systems. For instance, perturbation theory has been employed to examine magnetized dust collapse [5], Newtonian gravity to study magnetized binary neutron-star mergers [6], post-Newtonian (PN) calculations to inspect the premerger dynamics [7], and full general relativity (reduced to $2 \mathrm{D}$ by symmetries) to analyze axisymmetric differentially rotating star collapse [8]. We study the merger in full, 3D general relativity with a framework able to study the fluid's behavior and extract the gravitational waves in the wave zone. Clearly this system is highly relevant in the mechanism underlying short gamma ray burst phenomena. However, since we do not include radiation transport our focus in the present Letter is on the gravitational-wave signature and the fluid's behavior in a scenario probing the most extreme possible influence of the magnetic field.

Magnetic fields can have a strong influence on the dynamics of stars by introducing a number of instabilities (see, e.g., [9]). These instabilities allow for energy exchange between the fluid and magnetic modes and can redistribute the fluid's angular momentum through different mechanisms (e.g., magnetic winding and braking, magneto-rotational instabilities), depending on the strength of the magnetic field and the typical dynamical time scales $[10,11]$. These effects can have a nontrivial effect on the (time dependent) multipolar structure of the source, and consequently on the resulting gravitational waves. A detailed understanding of magnetic effects in binary mergers and stellar collapse will provide valuable information not only for deciphering the observed gravita- 
tional waves, but they might also influence the design and tuning of future advanced detectors [12,13].

In the present Letter we investigate possible magnetic effects in general relativity by simulating a merger of two compact stars with and without the presence of a magnetic field. We study the merger in full, 3D general relativity with a framework able to study the fluid's behavior and extract the gravitational waves in the wave zone.

II. Overview of the numerical approach. - We model the neutron-star material using relativistic ideal magnetohydrodynamics (MHD), coupled to the full Einstein equations of general relativity to represent accurately the strong gravitational effects during the merger. Our numerical techniques for solving these coupled equations have been tested previously [14-17]. Some essential elements of our implementation include the following. We use a computational infrastructure that provides distributed adaptive mesh refinement (AMR) [17-19] with full subcycling in time, with a novel treatment of artificial boundaries [19]. The refinement regions are determined by the truncation error of the solution during the evolution. We apply refined boundary conditions at the outer boundary to ensure consistency of the solution over the computational domain $[16,20]$ and adopt high resolution shock-capturing methods together with divergence cleaning to control the nomonopole constraint [14]. Our methods to solve the Einstein equations [16,20] and MHD equations [14] are described elsewhere.

III. Physical setup. - To begin exploring possible magnetic effects in binary systems, we compare two different binary neutron-star mergers, one with a magnetic field and one without. All other initial data are identical. For the magnetized stars, we choose a particular case in which each star initially has a poloidal magnetic field antialigned with the orbital angular momentum. Because the stars' magnetic fields are themselves aligned, this configuration induces a magnetic repulsion as the stars approach. Strong field repulsion should impact the dynamics of the system, and our results are compared with PN results presented in [7].

We construct the initial data with two identical neutron stars with zero spin angular momentum. Each star has a mass of $M=0.89 M_{\odot}$, a radius of $16.26 \mathrm{~km}$, and a central density of $3.24 \times 10^{14} \mathrm{~g} / \mathrm{cm}^{3}$. The stars are initially separated by $60 \mathrm{~km}$ and are given initial tangential velocities slightly below the Keplerian value. We adopt a simple polytropic equation of state choosing $\Gamma=2$ (polytropic index $n=1$ ) to model stiff nuclear matter [15].

The poloidal magnetic field is calculated from the vector potential $A_{\varphi}=\varpi^{2} \max \left(P-P_{\text {vac }}, 0\right)$, where $P$ is the pressure, $\varpi$ the cylindrical radius, and $P_{\text {vac }} / c^{2} \simeq 10^{7} \mathrm{gm} / \mathrm{cm}^{3}$ [21]. The field is initially confined to the stellar interior, and its maximum magnitude is approximately $9.6 \times$ $10^{15} \mathrm{G}$. While this value is large for astrophysical stars (excepting magnetars), it is small compared to the gravi- tational binding energy of the stars. As the individual stars do not rotate differentially, the initial field remains essentially constant until the stars are close enough for strong interactions.

The initial data are evolved in a cubical computational domain given by $[-1540 \mathrm{~km}, 1540 \mathrm{~km}]$ in each direction with an AMR configuration having 7 levels of refinement for the finest resolved simulation (5 and 6 levels of refinement were used for comparison). The highest resolution region follows each star and has a grid spacing of $\Delta=$ $0.46 \mathrm{~km}$. We extract the gravitational-wave information and compute the strain $\left\{h_{+}, h_{\times}\right\}$. Observers are placed at three different radii from the center of mass, namely, $r=\{440,590,740\} \mathrm{km}$. These correspond to $r \simeq$ $\{5,7.5,10\} \lambda_{\max }$, where $\lambda_{\max }$ is the largest wavelength in the observed gravitational wave. The extraction takes place in the wave zone, which is evident by the excellent agreement among the computed waveforms.

IV. Results. - The evolution of both binary pairs progresses quite similarly. The stars' orbits shrink as energy is lost through gravitational-wave emission. As they merge, a bar is formed, which itself is unstable. The bar expels matter and collapses to form a differentially rotating, hypermassive star. While rotation initially supports the star, the continued loss of energy through wave emission causes it eventually to collapse, forming a black hole. Comparing these two mergers, however, we see that the magnetic field strongly influences the details and time scales of the collapse, resulting in a solution that is clearly distinguishable from the unmagnetized case.

Initially, the two solutions are nearly identical. When the stars begin to disrupt and shed matter, initiating the merger phase, the aligned magnetic fields of the stars repel each other, delaying the final merger by 1-2 rotation periods with respect to the unmagnetized case. This delay is in agreement with post-Newtonian results presented in [7]. As the merger proceeds, a number of magnetically induced phenomena are observed [9-11]. First, a shear interface forms giving rise to Kelvin-Helmholtz instabilities, which are responsible for a rapid growth in the magnetic field strength, as shear energy is converted into magnetic energy (see Fig. 1). Second, differential rotation of the resulting merged star winds the magnetic field up, producing a toroidal field and linearly amplifying it as the star rotates. This trend saturates on the order of an Alfvén time scale, which in our case is $t_{A} \simeq 20 P_{c}$, where $P_{c}$ is the rotational period of the star's core. In our simulation this period is about $P_{c}=0.735 \mathrm{~ms}$ (cf. [8]). Third, at least two types of Tayler instabilities are displayed [10,22]. One is responsible for inducing an $e^{i m \phi}, m=1$ perturbation [10]. The second one induces a magnetic buoyancy instability which sheds some portions of the star in low density regions (about $1 \%$ of the star's maximum density) which manifest in cavities that can be observed in the magnetic case but are absent otherwise. Finally, as the star collapses to a black 


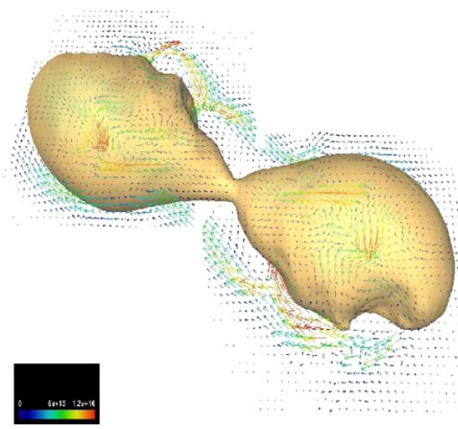

FIG. 1 (color online). Fluid density isopycnic (at $\rho=$ $6 \times 10^{13} \mathrm{~g} / \mathrm{cm}^{3}$ ) and magnetic field distribution (in a plane slightly above the equator) at $t=4.4 \mathrm{~ms}$. Kevin-Helmholtz instability in the shear layer has produced circulation cells that amplify the toroidal magnetic field. The cavities at both trailing edges are due to magnetic pressure inducing buoyancy.

hole, the magnetic field strength grows due to the compression of the field lines. The effects mentioned above strongly impact the fluid's distribution. Contrasting the magnetized case with the unmagnetized, Fig. 2 shows the ratio of the equatorial semiminor and semimajor axes while Fig. 3 shows density contours.

The combined effects of the magnetic field on the merger solution have a strong influence on the gravitational waveforms. Figure 4 shows the $l=2, m=2$ mode of $h_{+}$, the gravitational-wave signal. At early times before merger, the stars' magnetic fields do not strongly interact, and the waveforms are nearly identical. The delayed merger is clearly visible in the large amplitude of $h_{+}$which extends to $t \simeq 12 \mathrm{~ms}$, or $1-2$ more cycles than the nonmagnetized case. Magnetic effects in the post-merger waveform are also evident: $h_{+}$has both a smaller amplitude and lower frequency, especially for $t \gtrsim 20 \mathrm{~ms}$. The

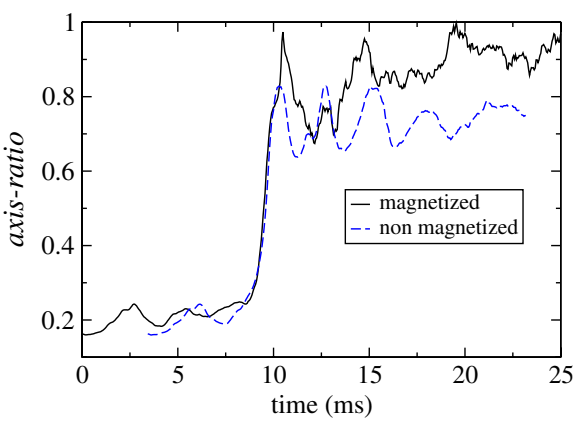

FIG. 2 (color online). Equatorial axis ratio (semiminor/semimajor) vs time for the nonmagnetized (dashed line) and magnetized (solid line) measured at a density of $1.24 \times 10^{14} \mathrm{~g} / \mathrm{cm}^{3}$. The results corresponding to the nonmagnetized case have been shifted in time by $t=3.5 \mathrm{~ms}$ so both mergers coincide. Notice how the magnetized case approaches the unit value much quicker while the nonmagnetized remains about $\simeq 0.78$. This translates into stronger amplitude in the produced gravitational waves.
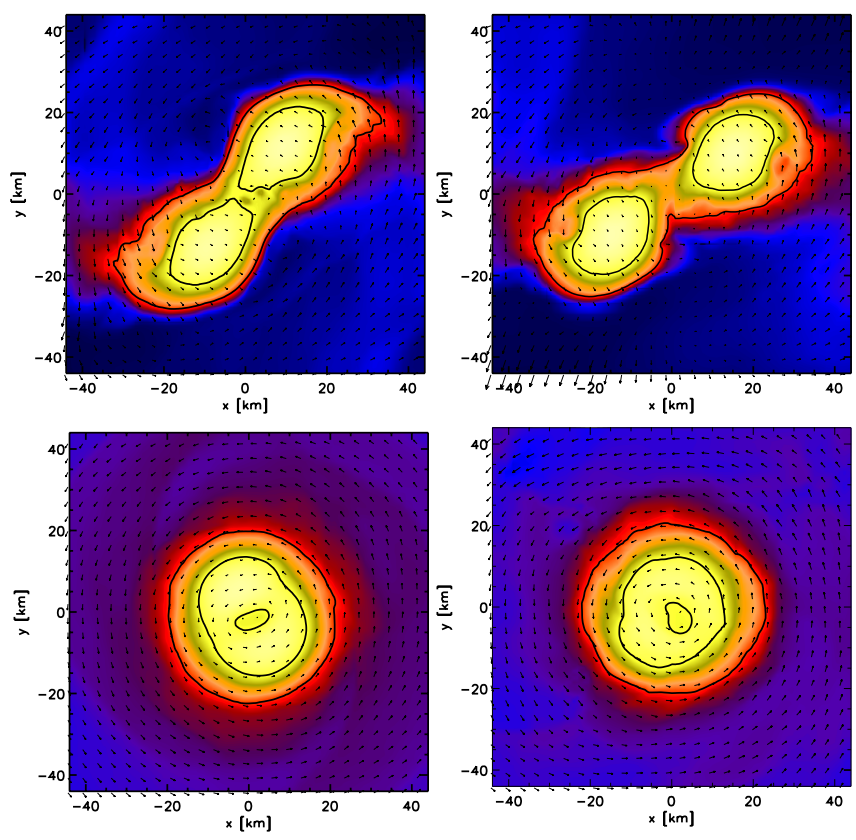

FIG. 3 (color online). Equatorial density contours and velocity field at 2 times for both the nonmagnetized (left column) and magnetized cases (right column). The top frames at $t=4.9 \mathrm{~ms}$ clearly show the delayed merger caused by magnetic field repulsion. The bottom frames are at $t=18.6 \mathrm{~ms}$. With no magnetic field, the final differentially rotating star displays a more barlike structure $(m=2)$, while the magnetized star is considerably more spherical with a slight offset.

magnetic field transports angular momentum away from the center, and as a result the rotation profile of the magnetized case displays a lower (higher) frequency at the inner (outer) regions of the star and a rather uniform rotation profile in between together with a more axisym-

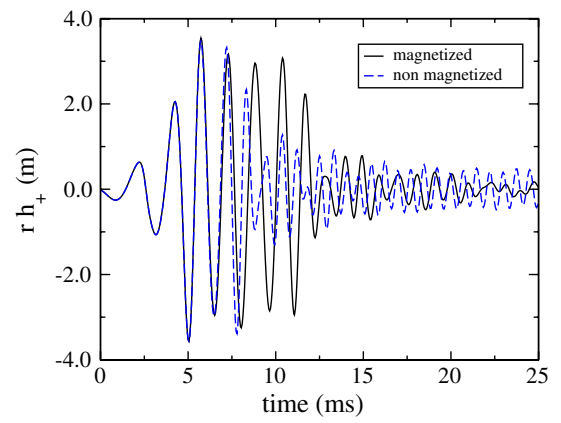

FIG. 4 (color online). Gravitational-wave signal $h_{+}(l=2$, $m=2$ mode) for the magnetized (solid line) and unmagnetized (dashed) mergers. Before $t \simeq 7.5 \mathrm{~ms}$, both signals are essentially the same. After $t \simeq 7 \mathrm{~ms}$, however, the delayed merger of the magnetized stars results in a much stronger signal lasting until $t \simeq 12 \mathrm{~ms}$. After merger, the redistribution of angular momentum by the magnetic field results in a more axisymmetric remnant whose core rotates more slowly. As a result, at late times the nonmagnetized merger produces radiation with larger (higher) amplitude (frequency) than its magnetized counterpart. 
metric configuration (see Figs. 2 and 3). Thus the core is more axisymmetric and has a longer rotation period in the magnetized case than in the nonmagnetized scenario. It is important to note that while our initial configuration has a large magnetic field, magnetic fields have been observed to saturate near this value in merger simulations that begin with weaker fields $\left(\simeq 10^{13} \mathrm{G}\right)$ [6]. The similarity in these post-merger field strengths and configurations leads us to believe that the dynamics will demonstrate analogous behavior. Finally, the frequency range of the obtained waveform is about $2.7-6.4 \mathrm{kHz}$. LIGO is sensitive to strains of $\simeq 4 \times 10^{-22} / \sqrt{\mathrm{Hz}}$ in this range [1]. The waves shown in Fig. 4 have amplitudes comparable to this noise at distances of 2 (4) Mpc for the nonmagnetic (magnetic) cases. Advanced LIGO would be able to detect these sources 10 times farther, including the Virgo cluster.

$V$. Conclusion. - We have presented a simulation of magnetized neutron stars with full 3D general relativity (GR) and MHD with sufficient resolution to capture key magnetically driven phenomena. We observe the transfer of shear energy into magnetic energy and magnetic winding, which are responsible for the creation and amplification of a toroidal field in the resulting hypermassive star. The strong differential rotation of this star transforms the poloidal field into a toroidal field, the former decreasing by an order of magnitude. The toroidal field redistributes angular momentum, resulting in an inner core rotating more slowly than the nonmagnetized case. While some of these effects have been seen to varying degrees in recent models (2D-axisymmetric simulations in GR [21], 3D smoothed particle hydrodynamics simulations without GR [6]), our implementation allows us to observe the magnetic interactions during the merger, consistently accounting for the tightening of the orbit due to the emission of gravitational waves, and does not restrict magnetic effects due to symmetries. The merger delay reported here has a strong influence on the resulting waveforms, since they have more relatively high-amplitude cycles. Additionally, the subsequent waveform displays observable differences in amplitude and frequency due to different magnetic instabilities. In our results, the inspiral (chirp) phase displays no significant difference but the merger and after-merger epochs do. In fact, a cross correlation between the two waveforms is equal to $0.98,0.63$, and 0.59 , when integrating from $t=0$ to $t=7.5, t=12.5$, and $t=$ $25 \mathrm{~ms}$, respectively. Searches for these types of magnetic effects in gravitational waves can give (upper-limit) population estimates of these and related binaries. Much more work will be required to map out the possible configuration space of field strengths and orientations, but the tantalizing differences observed indicate interesting results can be obtained and linked to gravitational and electromagnetic observations.

We would like to thank L. Bildsten, O. Blaes, J. Frank, G. Gonzalez, J. Pullin, I. Olabarrieta, and O. Reula for stimulating discussions. This work was supported by the NSF under Grants No. PHY-0653369, No. PHY-0653375, No. AST-0407070, and No. AST-0708551 to LSU, No. PHY-0326378 and No. PHY-0502218 to BYU, and No. PHY-0325224 and No. PHY-0643004 to LIU. Computations were done at BYU, LONI, LSU, and TeraGrid.

[1] R. E. Frey et al., AIP Conf. Proc. 928, 11 (2007).

[2] B. Willke et al., Classical Quantum Gravity 24, S389 (2007).

[3] F. Acernese et al., AIP Conf. Proc. 794, 307 (2005).

[4] R. C. Duncan and C. Thompson, Astrophys. J. 392, L9 (1992).

[5] H. Sotani, S. Yoshida, and K. D. Kokkotas, Phys. Rev. D 75, 084015 (2007).

[6] D. J. Price and S. Rosswog, Science 312, 719 (2006).

[7] K. Ioka and K. Taniguchi, Astrophys. J. 537, 327 (2000).

[8] M. D. Duez, Y. T. Liu, S. L. Shapiro, M. Shibata, and B. C. Stephens, Phys. Rev. Lett. 96, 031101 (2006).

[9] H. C. Spruit, arXiv:0711.3650.

[10] H. C. Spruit, Astron. Astrophys. 349, 189 (1999).

[11] A. L. Piro and L. Bildsten, Astrophys. J. 663, 1252 (2007).

[12] G. Harry, J. Houser, and K. Strain, Phys. Rev. D 65, 082001 (2002).

[13] I. Mandel, D. A. Brown, J. R. Gair, and M. C. Miller, astro$\mathrm{ph} / 0705.0285$.

[14] M. Anderson, E. W. Hirschmann, S. L. Liebling, and D. Neilsen, Classical Quantum Gravity 23, 6503 (2006).

[15] M. Anderson, E.W. Hirschmann, L. Lehner, S. L. Liebling, P. Motl, D. Neilsen, C. Palenzuela, and J.E. Tohline, Phys. Rev. D 77, 024006 (2008).

[16] C. Palenzuela, I. Olabarrieta, L. Lehner, and S. Liebling, Phys. Rev. D 75, 064005 (2007).

[17] S. L. Liebling, Phys. Rev. D 66, 041703 (2002).

[18] http://www.had.liu.edu/.

[19] L. Lehner, S. L. Liebling, and O. Reula, Classical Quantum Gravity 23, S421 (2006).

[20] O. Rinne, L. Lindblom, and M. A. Scheel, Classical Quantum Gravity 24, 4053 (2007).

[21] M. Shibata, Y. T. Liu, S. L. Shapiro, and B. C. Stephens, Phys. Rev. D 74, 104026 (2006).

[22] R. J. Tayler, Mon. Not. R. Astron. Soc. 162, 17 (1973). 